\title{
WikiRate.org - Leveraging collective awareness to understand companies' environmental, social and governance performance
}

- Richard Mills, Stefano De Paoli , Sotiris Diplaris, Vasiliki Gkatziaki , Symeon Papadopoulos, Srivigneshwar R. Prasad, Ethan McCutchen, Vishal Kapadia,Philipp Hirche

This is the authors' final version of an article published in Lecture Notes in Computer Science. The final publication is available at Springer via http://dx.doi.org/10.1007/978-3-319-45982-0 7 


\title{
WikiRate.org - Leveraging Collective Awareness to understand companies' Environmental, Social and Governance performance
}

\author{
Richard Mills ${ }^{1}$, Stefano De Paoli ${ }^{2}$, Sotiris Diplaris ${ }^{3}$, Vasiliki Gkatziaki ${ }^{3}$, \\ Symeon Papadopoulos ${ }^{3}$, Srivigneshwar R. Prasad ${ }^{1}$, Ethan McCutchen ${ }^{4}$, Vishal \\ Kapadia $^{5}$, and Philipp Hirche ${ }^{5}$ \\ 1 Department of Psychology, Cambridge University, England \\ 2 Abertay University, Dundee, Scotland \\ 3 CERTH-ITI, Thessaloniki, Greece \\ 4 Decko Commons e.V., Germany \\ 5 WikiRate e.V., Berlin, Germany
}

\begin{abstract}
WikiRate is a Collective Awareness Platform for Sustainability and Social Innovation (CAPS) project with the aim of "crowdsourcing better companies" through analysis of their Environmental Social and Governance (ESG) performance. Research to inform the design of the platform involved surveying the current corporate ESG information landscape, and identifying ways in which an open approach and peer production ethos could be effectively mobilised to improve this landscape's fertility. The key requirement identified is for an open public repository of data tracking companies' ESG performance. Corporate Social Responsibility reporting is conducted in public, but there are barriers to accessing the information in a standardised analysable format. Analyses of and ratings built upon this data can exert power over companies' behaviour in certain circumstances, but the public at large have no access to the data or the most influential ratings that utilise it. WikiRate aims to build an open repository for this data along with tools for analysis, to increase public demand for the data, allow a broader range of stakeholders to participate in its interpretation, and in turn drive companies to behave in a more ethical manner. This paper describes the quantitative Metrics system that has been designed to meet those objectives and some early examples of its use.
\end{abstract}

\section{Introduction}

Companies are increasingly expected to, and in some cases legally required to, report on their Environmental, Social and Governance (ESG) performance. The voluntary production of Corporate Social Responsibility (CSR) reports is now commonplace among large companies. Driving this trend is increased stakeholder demand [1], including demand from consumers [7]. Additionally, recently or forthcoming legislation requires:

- companies that trade in the United States and file with the Security and Exchange Commission (SEC) to produce "Conflict Minerals Reports" [22]. 
- companies that trade in the UK to publish statements about the steps they take to avoid slavery in their supply chains [15]

- Indian companies over a certain size to spend $2 \%$ of their profits on CSR activities [19].

- companies based in the EU with more than 500 employees tp report on ESG performance [10].

The majority of CSR reports are delivered as PDF documents (and/or online "Integrated Reports") following a bespoke structure as determined by the reporting company. The company has full control over this document, and freedom to present itself in the best possible light. One of the benefits companies seek when they engage in voluntary CSR reporting is an improvement in their reputation [4]. CSR reports tend to be written in a way that maximises this gain, and in some cases present disinformation or "greenwashing" [14].

To analyse a company's ESG performance based on their reporting, one must first interrogate that reporting and extract concrete information, then contextualise it by, for example, comparing to other companies of a similar size and/or operating in the same industry. This is however a difficult task, as it involves picking the same pieces of information out of the reporting output of every company being assessed.

Wikirate is a Collective Awareness Platform for Sustainability and Social Innovation (CAPS) project [23] funded by the Framework Programme 7, and launched in October 2013 with a mission to design and build a platform for "crowdsourcing better companies". This papers presents research conducted to determine how the WikiRate project could best pursue that goal, and the resulting design which deploys wiki principles to create an environment where the peer production[2] of data on ESG performance can take place. WikiRate offers a public repository where this data can be stored and tools for analysis and critique. Stakeholder demand is often cited as a driver of improved reporting [1], and WikiRate aims to demonstrate and increase the demand for this data by making it available in a usable format. As WikiRate is a peer production effort, all contributors are of equal status and can engage fully in the discourse about what we really want from CSR and the reporting thereof.

\section{Corporate Social Responsibility Reporting}

One of the major developments in CSR reporting in recent decades has been the establishment of reporting standards, and the adoption of these standards by many large corporations. The Global Reporting Initiative's (GRI) G3, and more recently G4, standards have the greatest levels of adoption by companies. The G4 defines 58 General Standard Disclosures, and 91 indicators for measuring sustainability impacts. The G4 guidelines state that companies should report on all of the 91 sustainability indicators that they deem "material" (relevant) to their business.

The degree to which companies disclose the information these indicators ask for varies between indicators, industries and companies. Sutantoputra [28] proposed a "social disclosure rating system" for assessing companies' CSR reports. This is based on the GRI's G3 guidelines and 
awards points based on whether a company reported specific pieces of information, giving more weight to "hard" indicators (where firms could face litigation if they are found to be lying) than "soft" indicators (which tend to be promises about the future). The rationale for this approach is Voluntary Disclosure Theory [27], which posits that while disclosures are voluntary, companies who perceive themselves to be performing well have an incentive to disclose more about their ESG performance.

Corporate Knights Capital [8] analysed the CSR reporting of the world's largest 4,609 listed companies in 2012 to see if seven sustainability indicators were disclosed. These indicators are as follows: Employee turnover: $12 \%$; Energy use: 40\%; Greenhouse Gas emissions: 39\%; Injury Rate: 11\%; Payroll: 59\%; Waste: $23 \%$; Water: $25 \%$. For a member of the public that is interested in the relative injury rates at competing companies in an industry, there is an $11 \%$ chance that they will be able to find information somewhere inside the CSR report of each company they research. These documents are often large, and finding the answer to a specific question involves looking up the indicator in an index (where this is provided) and then scanning a page/section to find the information.

In Corporate Knights' report [8] the seven indicators were each listed with GRI specification points, but also "Bloomberg ESG Fields". Bloomberg offer access to data about companies' ESG performance as part of their "Bloomberg terminal" service[3] which seems to cost around $\$ 24,000$ per year for a single terminal access point[21].There are several high-profile social and environmental ratings that are similarly opaque and inaccessible to the public ${ }^{6}$. There is evidence that these kinds of ratings can affect companies' behaviour. Chatterji [6] analysed companies that were covered by the KLD Social Ratings and found that companies with the worst performance in a year showed greatest improvement in subsequent years, more improvement than initial good performers and companies that weren't rated. Sharkey and Bromley [24] explored this further and reported an additional indirect effect whereby an increased number of rated peers led to reductions in toxic emissions, even among companies that were not themselves rated.

Some platforms, like CSRhub.com, mix data from paid-for and public sources together to produce a web-based offering that shows paying subscribers ratings of ESG performance broken down into themes - with an option to see values collected from public but not private sources. There are also ratings based on this data that are published openly ${ }^{7}$, with a description of their scoring methodology that explains it quite clearly. However, ratings based on private-access sources have an in-built limitation on how transparently they can present their scoring methodology it is difficult to expose the gears of the mathematical apparatus at work without also exposing the data being processed to produce each score. A useful quantitative record of companies' ESG performance is available for analysis, but only to people who are part of an organisation that can

\footnotetext{
${ }^{6}$ e.g. Kinder, Lydenberg, Domini Research \& Analytics (KLD) Social ratings, Dow Jones Sustainability indices

${ }^{7}$ Corporate Knights Capital also produce Newsweek's Green Rankings - http://www. newsweek.com/green/worlds-greenest-companies-2014
} 
afford access to this resource. This data is a commercial product, the raw material of a CSR analysis industry that uses it to produce reviews and ratings, and sells these products on to investors, analysts, and in some cases ethically minded consumers. Many stakeholders have no access to analysable data on companies ESG performance or proprietary ratings built upon that data. This limits the public's capacity to critique the actions of corporations and the manner in which these are reported. The data from CSR reporting is openly published, but it is also locked away either inside a PDF file or behind a paywall with an expensive key.

The GRI has already moved to encourage machine-readable CSR reports by developing and releasing an XBRL taxonomy - XBRL is a XML-like format used for much mandatory financial reporting. XBRL adoption was not immediately achieved in financial reporting due to organisational constraints such as legacy systems for reporting being perceived as delivering the same functionality [9]. This may also be a factor hindering a more widespread adoption of the standard in CSR reporting, and only a few examples of reports in this format can be found ${ }^{8}$.

Reporting standards like G4 are valuable because they ask concrete standardised questions of companies and can actually elicit responses. There are many questions about companies' ESG performance that can only be answered from within, and thus a company's reporting output is the original source for much of what is known about their performance. Reporting standards offer a framework for interpreting this output, posing a set of questions of companies' performance that either have answers or do not.

CSR reporting standards and procedures are still maturing, and issues like a lack of external assurance [11] remain to be resolved. Reading companies' reporting output is by no means the only way that the public can understand their impacts. There are many organisations taking a more active approach to investigating companies' behaviour. For example organisations like the Business and Human Rights Resource Centre ${ }^{9}$ collect and interpret qualitative information about companies' behaviour from pre-existing external sources.

Organisations like Amnesty International and Global Witness conduct investigative research that tends to focus on particular themes, using methods like interviews, secret filming/photography, and close scrutiny of documents/accounts ${ }^{10}$. The purpose of this research is to establish an evidence-base which can be used for advocacy - the focal point being a published report, with a campaign organised around that report. This kind of research sometimes involves the collection of useful company-level data as a by-product. For example, as part of the "Digging for Transparency" report into conflict minerals, Global Witness and Amnesty International[13] analysed Conflict Minerals Reports ${ }^{11}$ of 100 companies

\footnotetext{
8 https://www.globalreporting.org/services/Analysis/XBRL_Reports/Pages/default. aspx

9 http://business-humanrights.org/

10 https://www.globalwitness.org/en/about-us/

11 mandatory for companies that file with the United States Securities and Exchange Commission (SEC) under the 2010 Dodd Frank Act
} 
and found that 79 failed to meet the minimum requirements of the law. The reporting status of these 100 companies was not published as that was not the main thrust of the report, which sought to draw attention to a systemic problem. WikiRate offers a place to display this kind of data transparently and make it available for analysis by others, who can also critique or refine the methodology, and if they find it useful apply it to produce data for additional companies.

A number of organisations (e.g. Oxfam, Greenpeace) also produce public ratings of corporate performance along certain themes. In conducting this research, these organisations first define a set of indicators through which they measure companies' performance, then collect data to establish how companies perform in relation to those indicators, and produce a formula that turns the raw indicator data for a company into a score. The level of detail provided about the data and scoring methodology varies between projects. Oxfam's Behind the Brands ${ }^{12}$, and Ranking Digital Rights' Corporate Accountability Index ${ }^{13}$, are both noteworthy as being transparent with regard to the raw indicator data for companies and how this is turned into a score. Even in these cases however, because each organisation is conducting their research independently, and there are no common standards for how to define indicators or represent the data and scoring methodology - it is difficult for other researchers to replicate and build upon this work. The lack of commonalities in how this research is conducted and reported, and the fact that it is distributed between many different sources, makes it difficult for an individual to form a coherent understanding of how companies perform. The fragmentation of this research is also likely to diminish its potential to influence the behaviour of companies.

It is worth noting that there is a strong disconnect between the kinds of ratings that are offered to the public by organisations like Oxfam and Ranking Digital rights, and proprietary ratings such as KLD. NGOs may not have access to analysable data representing companies' reporting output, and proprietary indices may under-utilise publicly available data [5]. Knowing how a company is rated by KLD is restricted to those who have access, and to our knowledge it is not possible to see what the individual indicator values are for a company, or how those have been transformed into a rating. The public has limited insight into these ratings that can influence companies' behaviour [6], and the organisations producing these ratings not accountable to the public.

\section{WikiRate Core Concepts}

WikiRate is designed as a commons for the peer production[2] of an information resource that can be used to collectively: 1) establish what the impacts of a corporation's activities are and understand which practices or policies are causal; 2) identify the types of data that can be used to track companies' performance; 3) figure out which questions are most

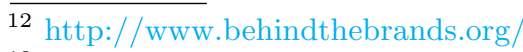

13 https://rankingdigitalrights.org/index2015/ 
important to ask; 4) find the answers to those quesions where they are available and 5) push companies to disclose them when they are not available.

Neutrality is one of the key principles around which the platform has been designed. WikiRate is not pro or anti-companies, and does not take a position on the relative importance of issues associated with corporate impacts. The only issue WikiRate takes a position on is corporate transparency. The manner in which companies behave and associated "externalities" should be out in the open for all to see. Without this information, it is impossible for stakeholders to form an accurate impression of which companies have net positive or negative impacts. WikiRate wants to enable stakeholders to formulate their judgments in an informed manner, not to dictate the relative importance of issues or metrics of performance.

Neutrality is important for WikiRate because ultimately the best source of information about companies' ESG performance should be the companies themselves. By being fair to companies and distinguishing between hard facts and value judgments, WikiRate offers companies a single place where they can organise and conduct their reporting in future, in a more direct and real-time dialogue with their stakeholders. The lag on CSR reporting has been identified as an issue with using this as an effective monitor of companies' behaviour[26], and WikiRate is well placed to mitigate this issue by allowing companies to enter data on a piece-by-piece basis as it becomes available.

WikiRate's design calls for two broad types of Metric. Researched Metrics are containers for storing "raw data" that comes from an external source, every value for these metrics must cite at least one source. Calculated Metrics serve the analysis of that data, they perform some mathematical or logical operation on input metrics to produce their output automatically. This fundamental distinction allows for the disassociation of data from analysis, and the easy re-use of data in multiple analyses that are free to interpret it in different ways. Metrics are complemented by structures for textual wiki content (the Wiki part of WikiRate, comprised of Notes and Reviews) that serve interpretation, critique, and information that does not fit neatly into Metric containers. Figure 1 shows a schematic overview of the relationships between WikiRate content types.

\subsection{Researched Metrics}

Researched Metrics have been designed to accommodate many different types of information in a standardised format, including

- low-level numerical indicators like quantity of water used

- binary or categorical answers to questions such as whether a company engages in a particular practice or not

- pre-existing ratings of company performance as produced by external research, advocacy and media organisations

A Researched Metric is a container for asking the same question of many different companies. Metric Values (data-points) represent the answer to that question for a particular company in a particular year. Metrics must 


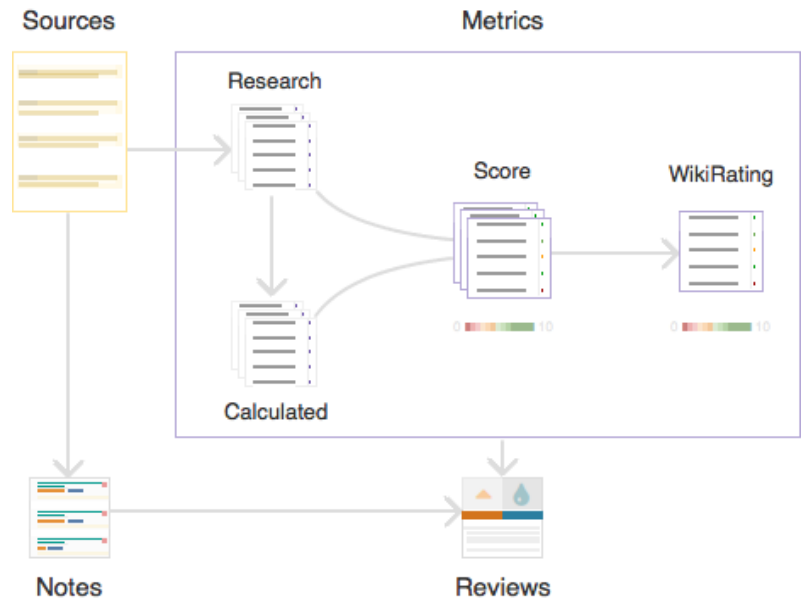

Fig. 1. Overview of the relationships between WikiRate content types

also have a short title that can be displayed in lists. Each metric must nominate a Metric Designer - this is the individual or organisation who formulated the question and defined a methodology for answering it $^{14}$. On the page for a company (figure 2) a reader can see all of the relevant metrics and the most recent answers for the company.

Metric pages (figure 3) display all of the meta-data associated with a metric, and show how a filterable selection of companies perform. There are two spaces for expanding upon the definition of a metric. The About section describes the information being sought, its utility, and/or how it should be interpreted. The Methodology section instructs researchers on how/where they can find the answer for a company.

Everything on WikiRate can be discussed and edited, to facilitate a discourse about how information should be interpreted and which questions are most important. Researched Metrics are containers for collecting raw data, but they also incorporate social spaces for critique and interpretation of that data. All researched metric values must have a source, and a reader can easily follow links to see these sources. An individual value for a researched metric has the following properties:

- the answer to the question the metric asks

- the company and year it relates to

- the source of the information

Finally, each researched metric has a Research Policy that determines who is eligible to add new values. Metrics that invite community members to research and add new values have a "community assessed" policy - these metrics tend to involve interrogating published documents like CSR reports or Conflict Minerals Reports to extract the answers to their question. Metrics that represent information like scores or ranks

${ }^{14}$ A Metric's full name is of the format Metric_Designer+Metric_Title, this allows metrics that share the same name to exist independantly in the system 


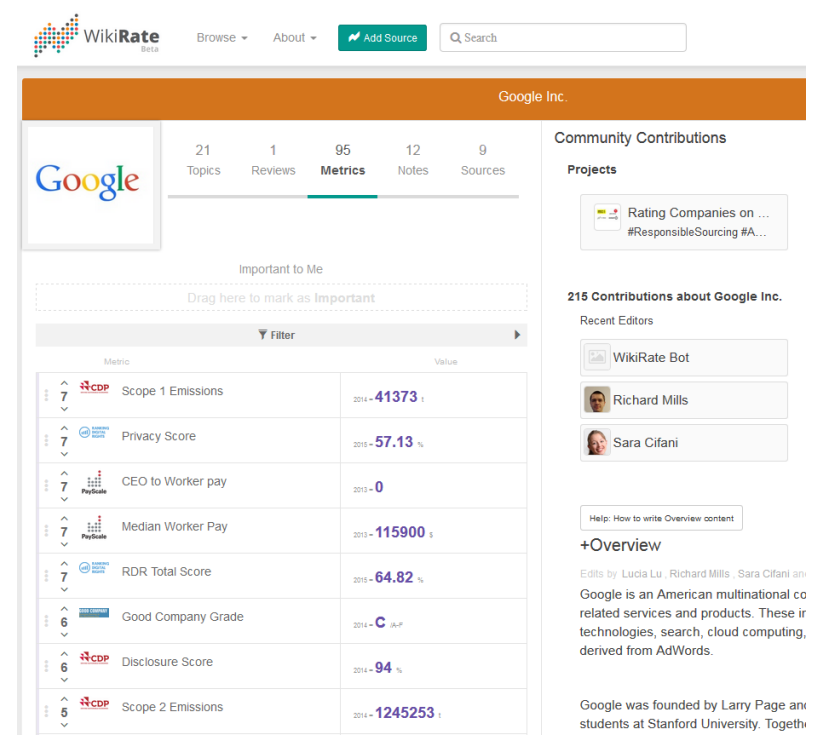

Fig. 2. The company page (Metrics tab) for Google Inc. on WikiRate

awarded by external entities have a "designer assessed" policy because only the designer is in a position to apply that scoring methodology faithfully to additional companies (or to cover additional years).

Researched Metric Data There are three ways in which WikiRate is gathering data for researched metrics.

1. Organisations that already produce company-level data about ESG performance design metrics and import their data from CSV files.

2. There are many existing publicly accessible and structured sources of company-level data scattered across the websites of different organisations. We are scraping this data and importing it as metric values that each link back to the original source.

3. WikiRate participants are encouraged to research and add new metric values for companies by following the methodology of the Metric they want to research ${ }^{15}$.

The first two methods are incorporated in recognition of the fact that a considerable volume of information relevant to assessing companies' ESG performance already exists in structured sources - it would be inefficient to task community members with adding this information on a value-byvalue basis when it can be added in bulk at relatively little effort. Our approach is to reserve the time and effort of our community members for tasks which require a human touch.

${ }^{15}$ Companies can answer questions about their own performance directly through the same mechanism, but must declare that the account used to add this data is operated by an official company representative 


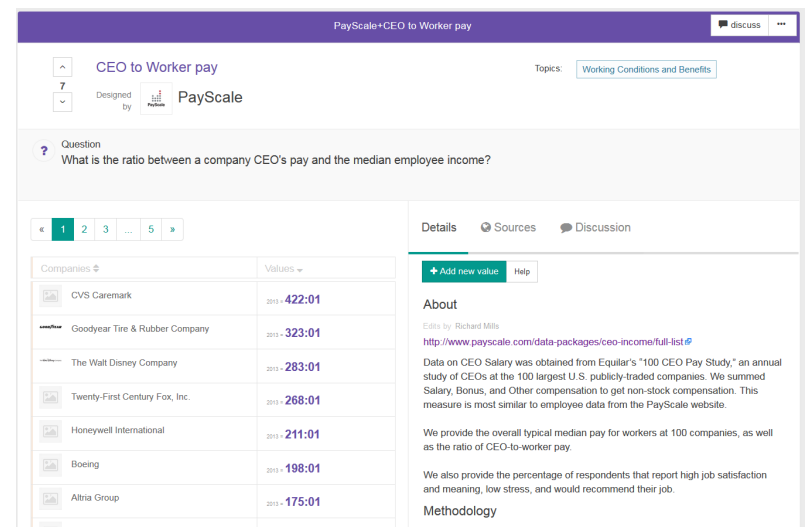

Fig. 3. The Metric page for PayScale+CEO_to_Worker_pay

Data Scraping An easy-to-use Information Extraction framework, named easIE, was developed to gather ESG data from publicly accessible Web sources and integrate it into WikiRate's database. easIE enables the extraction of ESG data about companies from heterogeneous Web sources in a semi-automatic manner, and organizes the extracted data around three key notions: (i) company: that represents a corporation (or a company) and is related with an id, name, country, a set of aliases and a website, (ii) metric: that represents a piece of information related to a company and (iii) article: the source of the information. A full description of easIE can be found in [12].

An extensive list of Web sources with CSR data was produced as part of they survey the ESG information landscape - by browsing the source lists of established aggregators of CSR data, and through communication with community members and other researchers/advocates. Next, each source was studied and the sources were ordered by the ease of extraction, the number of companies they covered and the relevance of the data they contained. Then, appropriate extraction rules for easlE were determined and a set of configuration files was built. So far, we have gathered data from 32 different sources. The created database comprises of 466,147 metric values related to 50,074 companies.

Peer Produced Data To facilitate the extraction of metric value data from unstructured source documents, an interface has been designed which emulates aspects of existing crowd-sourcing approaches (e.g. [20]). Source documents can be displayed in one part of the screen, and questions about the content of those documents are shown in the other. On one page the researcher can read the methodology for a metric, investigate whether the answer can be found in the available sources, and add the result of their investigation as a metric value. A series of related metrics can be strung together as part of a Project, so that researchers answer sets of questions whose answers are usually found within the same document. 
WikiRate is best described as a Peer Production[2] platform, all contributors are equal in principle and have permission to perform the same actions. This is a departure from many crowdsourcing initiatives, where "the crowd" is invited to perform certain tasks (like data capture or classification) but other tasks (like analysis of the resulting data) are reserved for the organisers of the initiative. User status on WikiRate is a social construct, established informally by one's contributions and how those are received by the community. The system is designed to function without designated gatekeepers or moderators.

One of the more difficult questions to answer when designing a peer production platform is "why will people be motivated to contribute?". Failing to find an answer to this question is the death of many such endeavours, and the only evidence that one has found the answer is a thriving community. At the moment, several hypotheses are being tested. NGOs with strong followings are increasingly sensing the potential of a closer engagement with their members. Amnesty International has a program dedicated to this - Alt-Clicktivism[16] seeks to "harness the power of the collective to shed light on human rights abuses worldwide". Where such NGOs have an interest in conducting company-level research into any aspect of ESG performance, WikiRate is well positioned as a platform where that research can be conducted openly. An NGO can design metrics that ask questions about companies' performance, offer guidance on finding the answers, and invite their members to participate directly in the research effort.

For students of sustainability or corporate behaviour, the task of interrogating a company's reporting output to find concrete answers to questions about their performance offers an interesting perspective on CSR. WikiRate's metric framing helps to highlight the information that is missing, and puts the information that's presented into context. WikiRate also offers an opportunity to complete an assignment that generates a public (by-)product which can be integrated into the research of others. Several metric research pilot projects with university course organisers have been established and are ongoing or due to commence soon.

Researched Metric Examples There follow some examples of how Researched Metrics are currently being used on WikiRate. Ranking_Digital_Rights+RDR_Total_Score ${ }^{16}$ is a percentage rating produced by Ranking Digital Rights:, it covers 16 major internet and telecommunications companies on 31 indicators and awards 3 sub-scores and a total score. PayScale+CEO_to_Worker_pay is a similar example of a metric based on external research that has been created in collaboration with the producers of the data.CDP + Scope_1_Emissions is a metric with values showing the number of tonnes of carbon (equivalent) emitted by 500 major companies, imported from the Carbon Disclosure Project's (CDP) public data-sets. This kind of data will make good raw material for calculated metrics related to climate change.

\footnotetext{
${ }^{16}$ Metric names are of the format Designer+Title, the URL for this metric is http: //www.wikirate.org/Ranking_Digital_Rights+RDR_Total_Score
} 
Amnesty_International+CMR_Lists_Smelters_and_Refiners is an example of a community-assessed metric. This metric was designed by Amnesty International as part of an ongoing edit-a-thon event pilot project. People are invited to attend and spend some time in groups researching companies to answer a set of questions about their conflict minerals reporting, and discussing what they find. Several other pilot projects experimenting with ways of generating different types of data are also ongoing. A set of metrics is being created to capture information related to GRI's G4 indicators in a way which is consistent with their XBRL taxonomy ${ }^{17}$. These are part of a pilot project inviting students of corporate sustainability to participate in liberating CSR reporting data from PDF files and making it available for analysis.

\subsection{Calculated Metrics}

Researched Metrics are containers for data about companies' behaviour, Calculated Metrics are designed to allow that data to be analysed transparently in public and in a modular fashion. There are three types of Calculated Metrics - Formula Metrics, Score Metrics and WikiRatings. Formula metrics will allow mathematical and logical operations to be performed on metric data. These metrics will make it easy to produce ratios or sums of metric values, but will also allow for complex calculations involving many steps. The Centre for Sustainable Organisations' context-based carbon metric ${ }^{18}$ has been identified as a challenging but achievable test case for a calculated metric. This is an open source metric that is currently available as a spreadsheet and used by some companies internally it calculates whether a company is emitting within its "allowance" of global emissions based on the RCP 2.6 scenario[25], using the company's economic output and greenhouse gas emissions as variables. The implementation of this metric on WikiRate will allow one to assess whether a company was emitting within its "allowance" of global emissions, once the input metric values have been added for a company this will be calculated automatically.

Score metrics will be used to add value judgments to a metric by mapping its range of possible values onto a 0-10 scale. The creator of a score metric imposes their opinions about what constitutes terrible/excellent performance by defining how values are mapped onto a 0-10 scale. The scoring approach will be slightly different for categorical and numerical metrics, but mapping different value types onto the same scale is a necessary step if we are to make all of this information available for inclusion in WikiRatings.

WikiRatings will calculate a weighted average for a number of Score metrics and produce a 0-10 score that measures how well companies perform on a theme defined by the designer. To design a WikiRating, a user selects a set of metrics that they want to include, selects or creates a

$\overline{17}$ Examples: Global_Reporting_Initiative+Environmental_fines_G4_EN29_a and Global_Reporting_Initiative+Collective_Bargaining_G4_11

18 http://www.sustainableorganizations.org/context-based-metrics-in-public-domain. html 
Score metric for each (that maps it onto a 0-10 scale), and then specifies the weight that each input metric should have. As all of the input metrics are on the same scale, the weight can be used as a direct indicator of the importance the metric designer places on the answer to each question. WikiRatings will offer an easy entry point to start comparing companies, because they embody an existing set of analyses that produce easily interpreted output. The primary consideration in the design of WikiRatings has been presenting them in the most transparent and understandable way possible. WikiRate wants to draw attention to the raw data that is being used in the calculations and show how each answer for a company contributes to a WikiRating.

\subsection{Metrics Marketplace and the WikiRate Index of Transparency (WRIT)}

With researched metrics to contain data, calculated metrics representing chunks of analysis, and every user being able to create metrics of any type, WikiRate's approach is likely to result in a large number of metrics ${ }^{19}$. To aid with the navigation of these proliferating metrics, WikiRate has the concept of a Metrics Marketplace powered by user preferences/votes. Any user can nominate metrics that are important to them (and these metrics will be displayed prominently for that user) or that they see no value in (and these metrics will be hidden from their view). These preferences also double as votes, the collective preferences for a Metric are used to produce a score that determines how visibly it is displayed. The metrics marketplace is designed to avoid a sprawling mass of undifferentiated metrics, it is a mechanism of determining what the most important metrics are and focusing the attention of readers (and companies) on those metrics. This is vital to facilitating better CSR reporting, because one of the complaints that companies make is that they are being asked to report too many different things by too many different entities [26]. The metric marketplace serves as a way of establishing what we care about collectively, which questions about companies' behaviour we most want to know the answers to.

The metrics marketplace will allow for the automated calculation of a "WikiRate Index of Transparency" (WRIT) score for each company, this will be the only Metric that WikiRate designs and endorses directly. WRIT will work by producing an importance score for every researched metric that takes into account both the direct importance votes on that metric, but also the importance votes of every calculated metric that uses it to perform some kind of calculation. Every metric will have a certain number of "transparency points" associated with it, determined by how heavily it is used within the system. A company's WRIT score will be determined by whether they have disclosed the answers to relevant researched metrics' questions. This will allow WikiRate to present companies with a list of questions for which we do not yet have their answers, and see how much their WRIT score could be improved by answering each question. WikiRate is developing a system of gamification

\footnotetext{
19 There are 290 metrics already available on the platform
} 
to incentivise user (and metric designer) participation [17] - the metrics marketplace and WRIT score can be thought of as an attempt to gamify CSR for companies.

\section{Conclusion and Future development}

WikiRate is designed to make the task of researching companies' ESG performance one that can be tackled collectively - breaking the process of defining indicators, collecting data and analysing that data down into granular tasks that can be completed by a range of actors in a collaborative space.

The broad goals of this collective research project are to:

1. collect the available information about companies ESG performance in one public place

2. see how much insight we can gain into companies' behaviour using this data

3. identify gaps or weaknesses with the available data, important questions that we cannot currently answer about companies

4. lobby for greater disclosure of that information

The major questions to address moving forward are whether people will be motivated to contribute to WikiRate (considered above) and whether their contributions will result in the high-quality information resource that is required to illuminate corporate impacts. WikiRate's approach to data quality is modelled on wiki principles, most content types can be edited directly by users, and everything has a full revision history showing who has edited it and what they have changed. Through discussion and direct editing, contributors to WikiRate are empowered to define and enforce standards for the evidence they want to collect. Testing whether these peer-produced researched metric values are accurate, and finding ways to improve both their quality and quantity, are some of the next priorities for research on the project.

It may be possible to find shortcuts to assuring the quality of data through the reputation of contributors. The creators and editors of every piece of content on WikiRate are prominently credited, and a user's profile page gives a detailed history of their participation on the platform. WikiRate's applies the principle of transparency to user activity in the same way that it is applied to the behaviour of companies. This extends to users' voting histories, which are a matter of public record on WikiRate - a departure from how up-down voting is usually deployed, and likely to influence the manner in which people vote[18].

This level of user transparency ${ }^{20}$ is important for WikiRate because of the nature of the subject matter. WikiRate is designed as a platform that can ultimately exert power over the behaviour of companies, this makes it a likely target for actors who want to distort how certain companies or themes are portrayed. Allowing users to see exactly what their peers are

${ }^{20}$ It is up to users whether they identify themselves or how they describe themselves, with the exception of users who have the authority to speak on behalf of companies or other registered organisations 
doing on the platform is necessary if the community is to self-moderate effectively. This level of transparency should also establish WikiRate as an interesting venue for research on how a collective awareness community behaves.

Acknowledgement: This work is supported by the WikiRate FP7 project, partially funded by the EC under contract number 609897 .

\section{References}

1. H. Aguinis and A. Glavas. What we know and dont know about corporate social responsibility a review and research agenda. Journal of management, 38(4):932-968, 2012.

2. Y. Benkler. The wealth of networks: How social production transforms markets and freedom. Yale University Press, 2006.

3. Bloomberg. Customers using esg data increased $76 \%$ in 2014 . http://www.bloomberg.com/bcause/ customers-using-esg-data-increased-76-in-2014. Accessed: 2015-0311.

4. S. J. Brammer and S. Pavelin. Corporate reputation and social performance: The importance of fit. Journal of Management Studies, 43(3):435-455, 2006.

5. A. K. Chatterji, D. I. Levine, and M. W. Toffel. How well do social ratings actually measure corporate social responsibility? Journal of Economics \& Management Strategy, 18(1):125-169, 2009.

6. A. K. Chatterji and M. W. Toffel. How firms respond to being rated. Strategic Management Journal, 31(9):917-945, 2010.

7. P. Christmann and G. Taylor. Firm self-regulation through international certifiable standards: Determinants of symbolic versus substantive implementation. Journal of International Business Studies, 37(6):863-878, 2006.

8. Corporate Knights Capital. Measuring sustainability disclosure: Ranking the world's stock exchanges. http://www.corporateknights. com/wp-content/reports/2014_World_Stock_Exchange.pdf. Accessed: 2015-03-11.

9. B. Doolin and I. Troshani. Organizational adoption of xbrl. Electronic Markets, 17(3):199-209, 2007.

10. European Commission. non-financial reporting. http://ec.europa. eu/finance/company-reporting/non-financial_reporting/index_en. htm. Accessed: 2015-03-11.

11. A. Fonseca. How credible are mining corporations' sustainability reports? a critical analysis of external assurance under the requirements of the international council on mining and metals. Corporate Social Responsibility and Environmental Management, 17(6):355370, 2010.

12. V. Gatziaki, S. Papadopoulos, Y.Tsampoulatidis, S. Diplaris, R. Mills, and Y. Kompatsiaris. Scalable analytics techniques for user contributions v2. Technical Report D5.5.2, WikiRate Project, September 2015. 
13. Global Witness and Amnesty International. Digging for transparency. https://www.globalwitness.org/en/campaigns/ conflict-minerals/digging-transparency/. Accessed: 2015-03-11.

14. W. S. Laufer. Social accountability and corporate greenwashing. Journal of Business Ethics, 43(3):253-261, 2003.

15. legislation.gov.uk. Modern slavery act 2015t, 2015.

16. M. Marin. How the wisdom of crowds can help defend human rights. https://www.amnesty.org/en/latest/campaigns/2015/ 10/wisdom-of-crowds-defend-human-rights/. Accessed: 2015-03-11.

17. R. Mills and S. D. Paoli. Interim report on user and community dynamics. Technical Report D3.3.3, WikiRate Project, September 2015.

18. R. A. Mills and A. Fish. A computational study of how and why reddit.com was an effective platform in the campaign against sopa. In Proceedings of the 2015 conference on Human Computer Interaction. Springer, 2015.

19. A. Prasad. India's new csr law sparks debate among ngos and businesses, Aug. 2014.

20. ProPublica. Free the files. https://projects.propublica.org/ free-the-files/sessions/new. Accessed: 2015-03-11.

21. Quartz. This is how much a bloomberg terminal costs. http:// qz.com/84961/this-is-how-much-a-bloomberg-terminal-costs/. Accessed: 2015-03-11.

22. SEC. Dodd-frank conflict minerals disclosure fact sheet. https: //www.sec.gov/News/Article/Detail/Article/1365171562058. Accessed: 2015-03-11.

23. F. Sestini. Collective awareness platforms: Engines for sustainability and ethics. Technology and Society Magazine, IEEE, 31(4):54-62, 2012.

24. A. J. Sharkey and P. Bromley. Can ratings have indirect effects? evidence from the organizational response to peers environmental ratings. American Sociological Review, 2014.

25. Stockholm Environment Institute. A guide to representation concentration pathways. https://www.sei-international.org/ mediamanager/documents/A-guide-to-RCPs.pdf. Accessed: 201503-11.

26. United Nations Environment Programme. Raising the bar.

27. R. E. Verrecchia. Discretionary disclosure. Journal of accounting and economics, 5:179-194, 1983.

28. A. Widiarto Sutantoputra. Social disclosure rating system for assessing firms' csr reports. Corporate Communications: An International Journal, 14(1):34-48, 2009. 